\title{
Magnetohydrodynamic Phenomena, Fluid Control and Computational Modeling in the Continuous Casting of Billet and Bloom
}

\author{
Shenqiang WANG, ${ }^{11 *}$ Gonzalo ALVAREZ DE TOLEDO, ${ }^{2)}$ Kari VÄLIMAA ${ }^{3)}$ and Seppo LOUHENKILPII) \\ 1) Department of Materials Science and Engineering, Aalto University, Vuorimiehentie 2K, FI-02150, Espoo, Finland. \\ 2) Gerdau Investigación y Desarrollo Europe, 48970 Basauri Vizcaya, Spain. \\ 3) Ovako Imatra Oy Ab, Terästehtaantie 1, Fl-55100, Imatra, Finland.
}

(Received on January 13, 2014; accepted on June 20, 2014)

\begin{abstract}
Magnetohydrodynamic flow phenomena with electromagnetic stirring were studied in billet casting. Solidification microstructure and oscillation marks were also investigated. EMS with $310 \mathrm{~A}$ current was proven to increase central equiaxed zone from $10-15 \%$ in unstirred billet to over $45 \%$ in stirred billet for steel grade 40CrMo4. Dendritic microstructures at the billet corners were found to deflect downwards due to the sustained upward flow along the mould corners. Rhomboidity was observed to relate to the unsteady flow in the mould during changes of casting speed and EMS intensities. Computational modeling was performed and validated with the results obtained from the casting experiments. Through modeling, a declining phenomenon of the injected stream from the SEN was observed towards the back wall under certain stirring intensities. This would influence thermal distribution of the strand shell and casting qualities, thus requiring correct matching of the vertical shear flow field with the horizontal rotation flow in order to obtain a favorable flow field. In addition, vortexes were found on the free surface around the SEN tube, which were influential to the cleanliness of steel melt. Modeling was also performed for a bloom caster with various SEN designs in the context of cleanliness. It was found that ported SENs showed obvious advantage on inclusion removal over the straight bore SEN. Reducing casting speed is actually an effective way to improve cleanliness. Casting trials with 65 bloom casting heats verified the modeling results and remarkable improvement in cleanliness was observed by using a 2-port SEN.
\end{abstract}

KEY WORDS: electromagnetic stirring; solidification microstructure; fluid flow; oscillation marks; inclusions; steel cleanliness; magnetohydrodynamics; computational fluid dynamics.

\section{Introduction}

During the 1970 's to 1980 's, there came a common understanding that increased solidification rate would reduce segregation and improve homogeneity of microstructures. Driven by the rapid expansion of market need and elevation of the downstream automation level, casting speed, i.e. productivity, had been universally increased on the continuous casting machines afterwards. Technically, the residence time of steel melt through the casting mould was greatly reduced. This brought new challenges for process control, defect prevention, and quality assurance in the continuous casting technologies. Fluid control techniques have been widely used to implement the target, including magnetohydrodynamic method, geometric method and thermophysical method, etc., assisted with computational fluid dynamic method.

Modern billet and bloom casting has ever stringent demands for product quality, meanwhile maintaining high productivity as well as low production cost. This requires better understanding and control of the casting process, including fluid flow phenomena, solidification microstructures, as well as various defect formation mechanisms. Especially, inclusion control has become a top important issue in the steel production worldwide, which is deeply involved in almost every aspect of steelmaking and casting

* Corresponding author: E-mail: shenqiang.wang@aalto.fi DOI: http://dx.doi.org/10.2355/isijinternational.54.2273 processes. Great effort sustained for over half a century has been put into cleanliness control in the various process stages from ladle to tundish till the casting mould and solidifying strand, and formed a comprehensive concept and technology called "clean steel" in the 1980's. ${ }^{1-3)}$ Even though, inclusions, including micro-inclusions $(<25 \mu \mathrm{m})$ and macro-inclusions $(>25 \mu \mathrm{m})$, originated from the upstream steelmaking and deoxygen processes will still be carried over into the casting stage and finally remained in the solidified microstructure. ${ }^{4}$ The remaining inclusions tend to exist interdendritically and intergranularly and may result in hot tearing and cracks during the solidification process. Also, internal and surface cracks may form in the downstream deforming and thermomechanical processes, finally deteriorating the mechanical properties, especially fatigue performances, of the component.5)

In addition to cleanliness control measures in the ladle and tundish, casting stage is the last opportunity to remove inclusions to the top slag layer which covers the steel melt in the mould. When casting engineering steel grades, a submerged entry nozzle (SEN) is usually applied to guide the steel melt from the tundish into the casting mould. Except for deoxidation products remaining in the steel melt there are other exogenous inclusions, typically reoxidation products, which can be very harmful and therefore should be removed from the melt and thus prevented from entering into the solidifying strand. Since the steel melt is the carrier of these inclusions, thereby, in order to implement inclusion control, fluid flow control in the mould is more significant. 
Nowadays, there are two approaches which are mostly used to implement fluid flow control. One is to impose an external force on the flowing fluid through magnetohydrodynamic techniques, e.g. electromagnetic stirring (EMS); another is to constrain geometrically the fluid flow in the mould via improved design and arrangement of mould, SEN and strand guiding segment to attain demanded cleanliness as well as favorable solidified microstructures.

In slab casting, a two-port design on the SEN end is the normal construction because of the flat geometry of the slab mould. In bloom and billet casting, straight bore metering SEN is normally used. A typical drawback of the straight bore SEN design is that melt stream penetrates deep into the liquid pool within the strand. Except for the thermal impact to the lower strand shell, the inclusions dispersed with the stream also have difficulties in floating up to the top slag to be removed., ${ }^{6,7)}$ Especially, with high casting speed, this problem becomes more serious. ${ }^{8)}$

Many studies have been carried out to investigate swirling SEN designs through various approaches. Sormann et al. ${ }^{7}$ developed a precision control valve (PCV) at the tundish exit, combined with a swirl SEN. The swirling flow was generated with an eccentric throttle slot in the piston of the PCV. The stream penetration depth was remarkably reduced and the meniscus wave diminished even at higher casting speed. Meanwhile, using rotational electromagnetic field to generate a swirling flow in the SEN was suggested. Yokoya and Kholmatov et al. ${ }^{9-11)}$ studied a swirling SEN with various divergent angles at the SEN end for square and round billets, where the swirling flow was created by swirl blades mounted at the upper end of the tube. The swirling flow enhanced dissipation of the melt superheat in the mould and remarkably reduced stream penetration depth so that the inclusions were favored for floatation. The present authors ${ }^{12)}$ studied the effect of a rotational electromagnetic field around the SEN tube (SEN-EMS) on the inclusion removal for bloom casting by computational modeling. The SEN-EMS clearly increased the removal rate of inclusions for various inclusion sizes, due to reduced stream penetration and lateral dissipation flow in the mould. However, considering the operational conveniences and cost, these approaches have not been widely used in the practice.

Bloom is different from billet by leaving much larger space in the mould for more complicated SEN design and thus for better control of the flow field and cleanliness. Pindor and Michalek ${ }^{13)}$ studied multi-port designs with 4 and 5 outlets, via mathematical and water modeling as well as casting trials. Finally they came to an "umbrella design" with a spherical cap to guide the upward 4 ports. The main idea is to distribute the melt stream sideways and upwards to the lateral mould walls in order to enhance the removal of inclusions. Their study indicated that immersion depth of the SEN was an important casting factor. The immersion depth between $200-240 \mathrm{~mm}$ gave the best results. The casting trials showed that the semis quality for carbon content less than $0.3 \mathrm{wt} \%$ was greatly improved, and product rejection rate was reduced from $1.39 \%$ to zero compared with the cast product using straight bore metering SEN.

In this paper, the two fluid flow control techniques, i.e. EMS and SEN design were investigated experimentally and numerically in billet and bloom casting. The influence of the fluid flow on initial solidification microstructure was studied by casting trials in a steel plant. The effect of EMS induced rotational flow on the meniscus shape and oscillation marks was also studied. SEN designs including 2-port designs with various guiding angles, a 4-port and a straight metering SEN were simulated and their effect on the inclusion removal was compared by using computational fluid dynamics modeling. In-mould electromagnetic stirring was simulated to evaluate the influence of EMS on the fluid flow and inclusion removal. The industrial casting trials with a 2port SEN were performed and compared with the results by the metering SEN.

\section{In-plant Casting Experiments about the Effect of EMS on Fluid Flow, Solidification Microstructure and Meniscus Shape}

\subsection{In-mould EMS Setups and Measurement of Mag- netic Field}

Experimental work for characterizing the effect of inmould EMS on the liquid flow was carried out at Gerdau Special Steels Europe Basauri plant. Figure 1 shows a sketch of the stirrer situated at the end of the mould cassette and measurement of the magnetic flux density along the mould axis for the $185 \mathrm{~mm}$ billet. It is seen that the maximum values of the magnetic field are situated at the stirrer centre, around $734 \mathrm{~mm}$ apart from the mould top. Near the meniscus, $150 \mathrm{~mm}$ distance from the mould top, the magnetic field value was $5 \%$ of the maximum value at the centre of the mould.

\subsection{Fluid Flow and Solidification Microstructure}

The liquid flow in the mould was evaluated based on the deflection of the primary dendritic arms in the microstructure. Even, comparison with other techniques used to measure liquid flow on the meniscus during casting was made. It is known that during solidification process in the mould, the primary dendrites will grow in a deflected direction opposite to the melt flows. Takahashi et al. ${ }^{14)}$ developed the following mathematical expression relating the degree of the dendritic deflection with the fluid velocity of the steel melt in the solidification front which is correspondent to the intensity of the local magnetic field:

$$
\theta=22.49 U^{-0.177} \log \left(3.72 \times 10^{-3} U^{2.08} / V\right)
$$

where $\theta$ is the deflection angle in degrees; $U$ the fluid velocity in $\mathrm{cm} \cdot \mathrm{s}^{-1}$; and $V$ the solidification front speed in $\mathrm{cm} \cdot \mathrm{s}^{-1}$.

Industrial trials to assess the effect of mould EMS on the solidification structure and steel cleanliness were also carried out. A heat of steel grade $40 \mathrm{CrMo} 4$ was cast with 3 different EMS settings of 0,150 , and 310 A electric current. Figure 2 shows the solidification structure and geometric quality of the billet with the defined casting parameters under the different EMS intensities. The EMS was switched off after 5 minutes of casting for 35 minutes then switched on and the electric current was raised up to $310 \mathrm{~A}$. After 57 minutes of casting it was decreased to 150 A of electric cur-

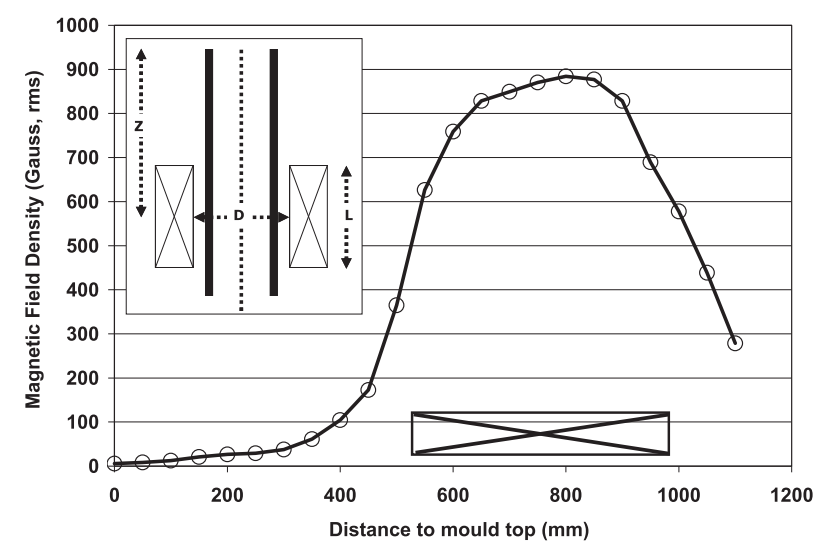

Fig. 1. Sketch of the mould stirrer situated at the lower part of the mould and variation of the magnetic flux density along the billet caster mould. Measurements were carried out at the Gerdau Special Steels Europe, Basauri plant for $185 \mathrm{~mm}$ square billets. 


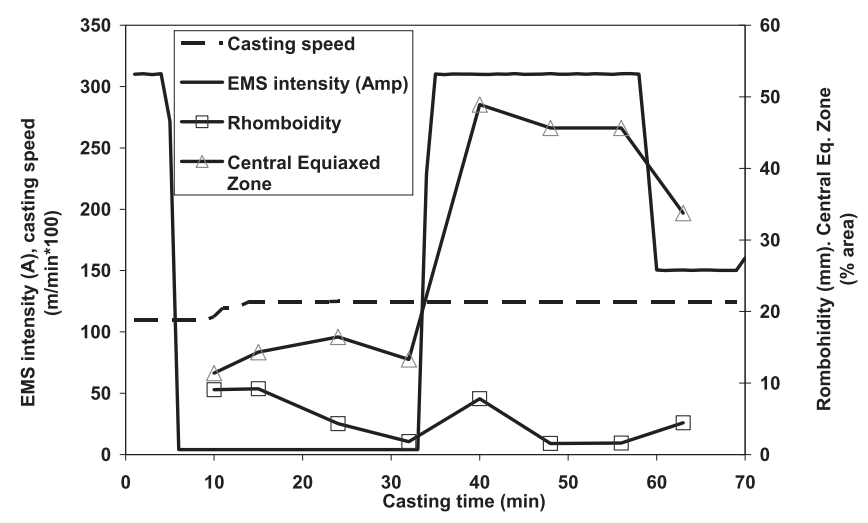

(a)

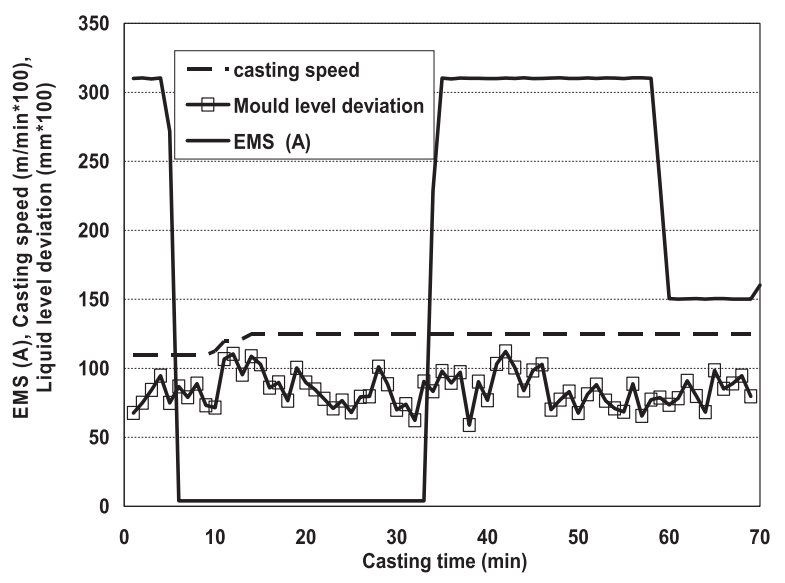

(b)

Fig. 2. Influence of the EMS intensities (0, 150 and $310 \mathrm{~A})$ during casting a heat of grade $40 \mathrm{CrMo} 4$ on casting parameters and billet metallographic characteristics: (a) Measured central equiaxed zone ratio and rhomboidity; (b) Meniscus level deviation (Gerdau R\&D)

rent. The melt temperature and casting speed remained constant during the testing period of time.

The squared marked line in Fig. 2(a) represents the changes of rhomboidity measured from the transversal samples of the cast billet. Four of the eight samples showed significant rhomboidities. The first two bigger rhomboidity values could be related to the increase of casting speed at the beginning of the test and the latter two high values might correspond to the changes in the mould EMS intensity during casting. It can be concluded that changes of flow field in the mould, i.e. the stirring intensity and casting speed, enhance rhomboidity, whereas casting under stabilized flow field produces better geometric quality of the cast billet. This finding agrees principally with Madias ${ }^{15)}$ that rhomboidity is generally attributed to non-uniform cooling during the initial shell formation in the mould.

The percentage area occupied by the equiaxed structure was measured from the transversal samples and is also presented in Fig. 2(a). It is clearly seen that by increasing the mould EMS intensity, the proportion of central equiaxed zone remarkably increased. The equiaxed crystal ratio (ECR) increased from $10-15 \%$ without stirring to over $45 \%$ with 310 A stirring intensity, and then down to $30-35 \%$ with 150 A stirring intensity.

\subsection{Near-meniscus Flow and Oscillation Marks}

The standard deviation calculated for 1 minute time period of the meniscus level, measured by the radioactive control system, can be seen in Fig. 2(b). It is noteworthy that no change was observed for the mould level stability by

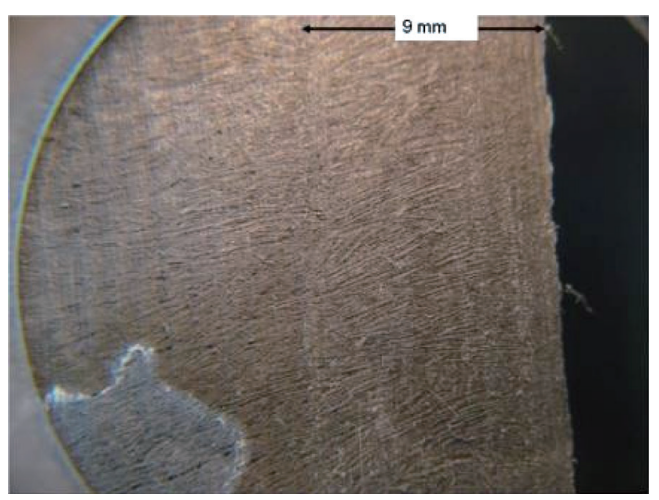

Fig. 3. A typical microstructure near the surface in which dendrites are seen tilted. This indicates that there is a rotational flow of the liquid steel near the meniscus agitated by the stirrer (Gerdau R\&D).

changing the mould EMS. This result suggested that if the mould stirrer was mounted far below the meniscus (the upper edge of stirrer coil was $470 \mathrm{~mm}$ from the meniscus), it had no significant influence on the meniscus level. However, this result seems opposite to the effect of the EMS on the deflection of dendrites just below the billet surface as shown in Fig. 3, where the microstructure near the surface for a sample was produced with 310 A EMS. It can be observed that the deflection of dendrites started from almost the billet surface. Using the Takahashi et al. expression, EMS induced flow velocity of $0.2 \mathrm{~m} / \mathrm{s}$ can be deduced when the dendrite deflected $21^{\circ}$. Some discontinuity in the dendrite growth is observed at $9 \mathrm{~mm}$ from the surface possibly because the solid shell reached the zone of the maximum magnetic field. These observations indicate that there was a rotational flow of the liquid steel near the meniscus agitated by the stirrer, but this rotational flow didn't bring about sensible disturbance to stability of the mould level. In order to clarify this apparent contradiction, some more metallographic studies and modeling were also carried out.

Oscillation marks are, in general, regarded as regularly spaced, transversal indentation defects and overlapping marks formed at the initial solidification stage of the shell wafers on the surface of continuously cast products due to mould oscillations and fluid instabilities in the liquid strand pool. ${ }^{16)}$ In order to study the influence of EMS on the meniscus solidification, i.e. oscillation marks, longitudinal slices with $15 \mathrm{~mm}$ thickness were cut from all the four faces of a $250 \mathrm{~mm}$ billet length sample. Figure 4 shows the photograph of all the four slices (marked 1-4). The off corner regions against the EMS induced liquid flow (flow hitting the wall) are marked with " $X$ ". Figure 5 presents the oscillation marks morphology by hot acid etching on the billet surface. Typical surface oscillation marks shown in Fig. 5(a) (for $40 \mathrm{CrMo} 4$ steel cast with 310 A EMS) indicate that the oscillation marks arose around $8-10 \mathrm{~mm}$ towards the billet corner, in the area which was against the liquid flow. Similar results were observed for all the other faces. Samples taken without mould EMS did not show such a rise in the oscillation marks at the corners. To further verify this phenomenon, longitudinal billet surface samples were also prepared from another heat. Figure 5(b) shows the oscillation marks on one surface of the billet produced with new composition (steel $\mathrm{C} 10 \mathrm{E}$ with $0.1 \% \mathrm{C}$ cast with $270 \mathrm{~A}$ MEMS). It can be seen that about $6 \mathrm{~mm}$ rise of the oscillation marks has occurred at the corner side against the liquid flow and similar rise for all the other faces as well.

\subsection{Vertical Upward Flow and Downward Deflection of Primary Dendrites at the Corners}

A metallographic analysis of the two end longitudinal 


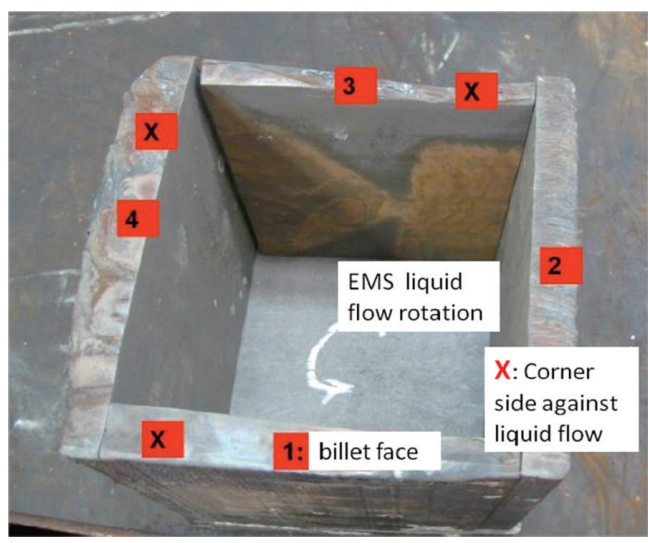

Fig. 4. Longitudinal surface slices for metallographic analysis to study the influence of mould EMS on billet surface quality and oscillation marks formation. "X" indicates the corner side against the liquid flow (Gerdau R\&D).

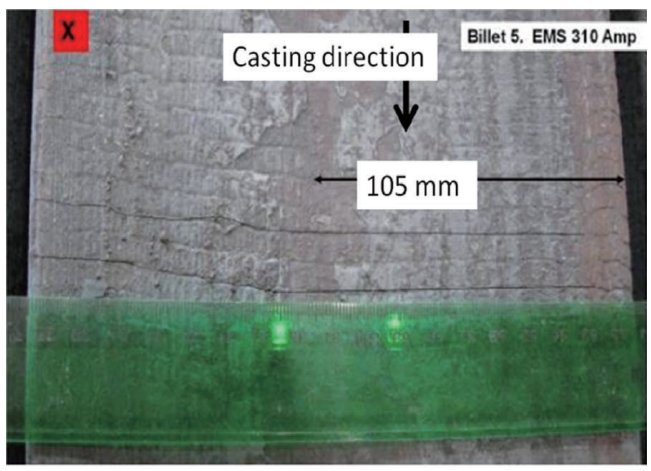

(a)

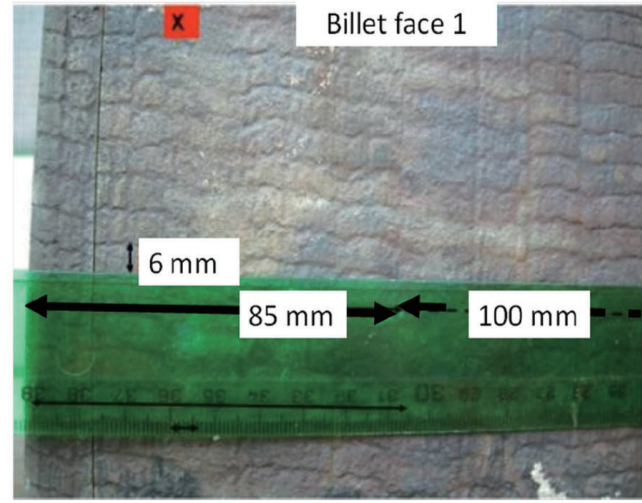

(b)

Fig. 5. Influence of EMS on the rising of oscillation marks at the corner side against the EMS induced rotational liquid flow, with view of the billet surface after hot acid etching. (a) Steel grade 40CrMo4, EMS current $310 \mathrm{~A}$; (b) Steel grade C10E, EMS current 270 A (Gerdau R\&D).

surfaces of billet face 1, in Fig. 4, showed an interesting result: dendrites were also deflected in one of the sample ends, and the deflecting direction indicated an upward fluid flow towards the meniscus at the corner side against the rotational liquid flow. The deflection angle was measured at about $18^{\circ}$ which corresponds to a vigorous liquid velocity of around $0.14 \mathrm{~m} / \mathrm{s}$ in this region calculated by Eq. (1). In order to investigate if the upward liquid flow exists all along the entire perimeter, one of the billet surface samples was cut in $20 \mathrm{~mm}$ wide slices, each of which was hot acid etched to reveal the primary dendrite morphology. Figure 6(a) shows the positions of the billet face (arrowed) where

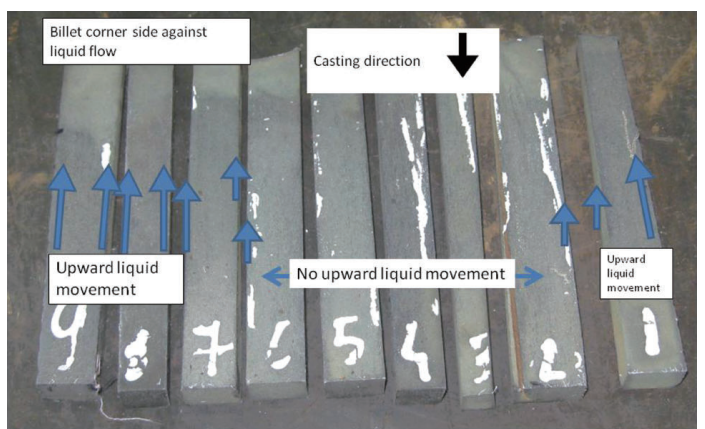

(a)

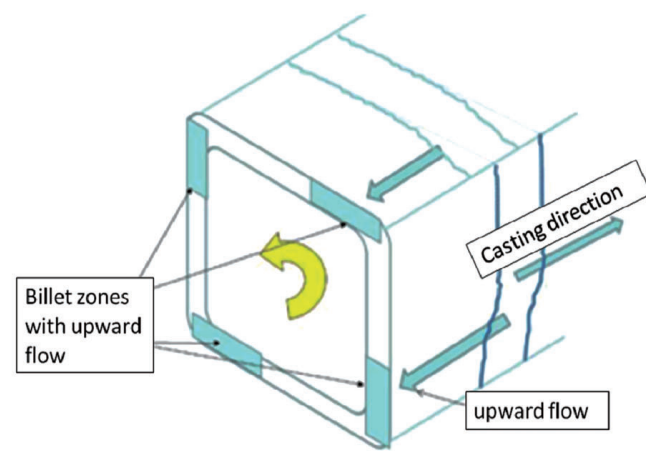

(b)

Fig. 6. (a) Observation and estimation of the longitudinal fluid flow based upon deflecting of the primary dendrite morphology in one of the billet faces of Fig. 4 billet surface samples. (b) A sketch of the relationship between EMS induced liquid rotation, liquid upwards flow and elevation of the oscillation marks (Gerdau R\&D).

upward flow was observed and the corresponding liquid flow velocity calculated from dendrite tilting measurements with Eq. (1) are displayed in Fig. 7(a). It seems that there was an upward liquid flow only at the corner sides which were against the rotational flow and this upward flow at the corners drove the oscillation marks at the meniscus area to rise and formed a waved configuration of the oscillation marks. This is diagrammatically demonstrated in Fig. 6(b).

In addition to the studies above on the EMS induced flow in the billet mould, casting trials were also carried out on a bloom caster at Ovako Imatra plant to study the effect of SEN designs on steel cleanliness, which is described in a latter section.

\section{Computational Modeling}

\subsection{Mould Setups of the Billet and Bloom Casters}

The setups of the billet mould at Gerdau R\&D and the bloom mould at Ovako are listed in Table 1, where the billet section is $185 \times 185 \mathrm{~mm}^{2}$ with an arc radius of $9 \mathrm{~m}$ and the bloom section is of the size $371 \times 311 \mathrm{~mm}^{2}$ with an arc radius of $15 \mathrm{~m}$. EMS stirring center is positioned $734 \mathrm{~mm}$ below the mould top for billet and $500 \mathrm{~mm}$ for the bloom. Figure 8 illustrates the different SEN designs for the bloom caster and the geometric details can been seen in Table 2, where (a) is a straight bore type; (b)-(d) are two-port types with various port geometries and guiding angles; and (e) is the four port type. The inner and outer diameters of the SENs are $\varnothing 55 \mathrm{~mm}$ and $\varnothing 108 \mathrm{~mm}$ respectively. For the ported SENs, (b) and (c) are of rectangular port with sizes of $62 \times 42 \mathrm{~mm}^{2}$ and $50 \times 48 \mathrm{~mm}^{2}$ respectively; (d) is of round port with a diameter of $\varnothing 55 \mathrm{~mm}$, which is the same as the inner diameter of the SEN tube; (e) is a combination of round and rectangular with an outer size of $ø 38 \times 55 \mathrm{~mm}^{2}$. For the guiding angle of the port, (b) and (c) are $30^{\circ}$ and $15^{\circ}$ 


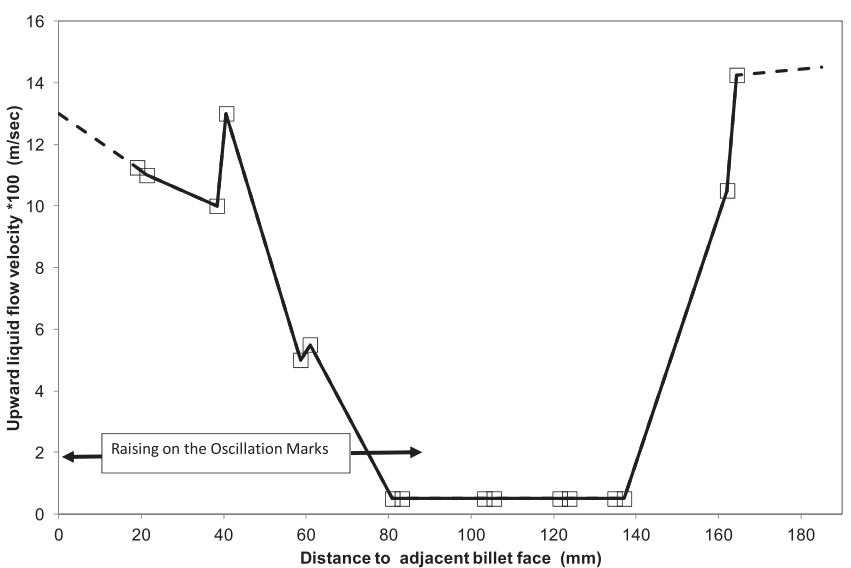

(a) Calculated vertical velocity by dendrite deflection measurement

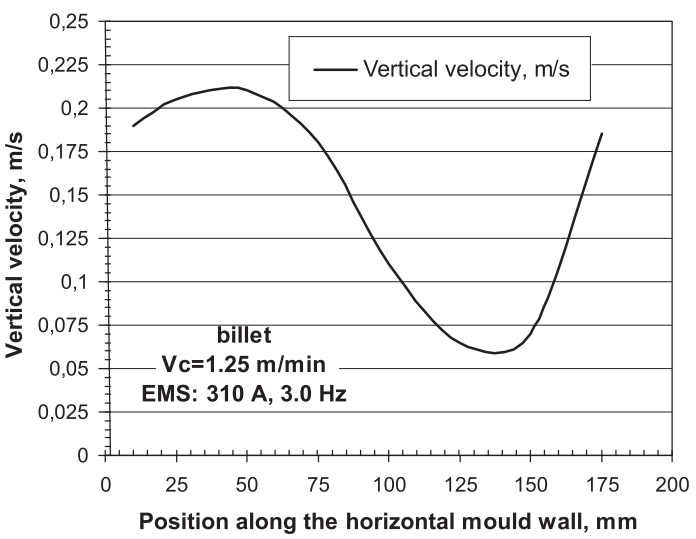

(b) simulated maximum vertical velocity above stirring center

Fig. 7. Longitudinal upward liquid steel velocity in the billet face shown at Fig. 6(a). The fluid flow velocity has been calculated using the Takahashi et al. ${ }^{14)}$ equation and the deflection measurements of the primary dendrites at different surfaces of billet slides (Gerdau R\&D) (a) and using CFD modeling (b).

Table 1. Mould-SEN-EMS setups of the billet and bloom.

\begin{tabular}{ccc}
\hline & Billet & Bloom \\
\hline Mould size, mm & $185 \times 185$ & $371 \times 311$ \\
Mould length, m & 1.0 & 0.7 \\
Strand bending arc radius, m & 9.0 & 15 \\
SEN diameter, mm & $\varnothing 36-46(\varnothing 73-76)$ & $\varnothing 55(\varnothing 108)$ \\
SEN immersion depth, mm & 110 & $150-180$ \\
Casting speed, m/min & 1.25 & 0.6 \\
EMS current, A & $0,150,310$ & $0,150,300$ \\
EMS magnetic flux, Gauss & $0,420,870(\mathrm{rms})$ & $0,870(\mathrm{rms})$ \\
EMS frequency, Hz & $3.0 \mathrm{~Hz}$ & $2.0 \mathrm{~Hz}$ \\
EMS stirring position, mm & -734 & -500 \\
\hline
\end{tabular}

downward respectively; (d) and (e) are $15^{\circ}$ upward. For the SEN immersion depth, (a)-(c) are $150 \mathrm{~mm}$ below the meniscus; (d) and (e) are $180 \mathrm{~mm}$ below the meniscus.

\subsection{Fluid Flow Models}

The steel melt flow in the mould was computed by applying the ANSYS-FLUENT CFD software. The turbulence of the flow was treated with the realizable $\mathrm{k}-\mathcal{E}$ turbulence model. Heat transfer was not considered in this simulation. The

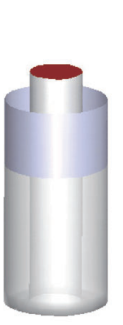

(a)

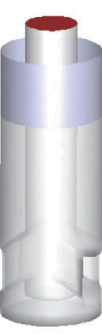

(b)

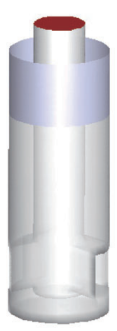

(c)

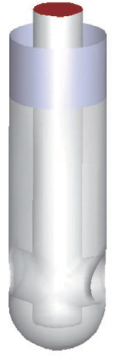

(d)

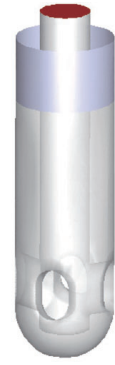

(e)
Fig. 8. SEN types (a)-(e) for the bloom caster (Ovako Imatra).

Table 2. Geometric details of the SEN designs for the bloom caster used in the modeling.

\begin{tabular}{cccccc}
\hline & $(\mathrm{a})$ & $(\mathrm{b})$ & (c) & (d) & (e) \\
\hline Number of ports & 0 & 2 & 2 & 2 & 4 \\
$\begin{array}{c}\text { Tube diameters, } \\
\text { mm }\end{array}$ & $\varnothing 55$ & $\varnothing 55$ & $\varnothing 55$ & $\varnothing 55$ & $\varnothing 55$ \\
Port size, mm & - & $62 \times 42$ & $50 \times 48$ & $\varnothing 55$ & $\varnothing 38 \times 55$ \\
$\begin{array}{c}\text { Guiding angle, } \\
\text { SEN immersion } \\
\text { depth, mm }\end{array}$ & -90 & -30 & -15 & +15 & +15 \\
\hline
\end{tabular}

boundary conditions were defined corresponding to the casting speed of $0.6 \mathrm{~m} / \mathrm{min}$. The physical properties were supposed to be constant: the density of molten steel was between 7000-7 $200 \mathrm{kgm}^{-3}$ depending on the steel grade and the density of slag $3500 \mathrm{kgm}^{-3}$; the viscosity of steel melt and slag was chosen 0.0063 and $0.065 \mathrm{~Pa} \cdot \mathrm{s}$ respectively.

The momentum balance equations are as follows:

$\rho \frac{\partial u_{i}}{\partial t}+\rho u_{j} \frac{\partial u_{i}}{\partial x_{j}}=-\frac{\partial p}{\partial x_{i}}+\frac{\partial}{\partial x_{j}}\left[\mu\left(\frac{\partial u_{i}}{\partial x_{j}}+\frac{\partial u_{j}}{\partial x_{i}}\right)\right]+\rho g_{i}+F$

where $\mu=\mu_{0}+\mu_{\mathrm{t}} . \mu_{0}, \mu_{\mathrm{t}}$, and $\mu$ are the molecular dynamic viscosity, turbulent viscosity, and effective viscosity $(\mathrm{Pa} \cdot \mathrm{s})$. $\rho$ is the density of steel melt $\left(\mathrm{kgm}^{-3}\right) . u_{i}, u_{j}$ are fluid velocities in the $i$ th and $j$ th directions respectively $\left(\mathrm{ms}^{-1}\right) . g_{i}$ is gravitational acceleration in the $i$ th direction $\left(\mathrm{ms}^{-2}\right) . p$ is the pressure in the fluid $(\mathrm{Pa}) . t$ is time (s). $x_{i}, x_{j}$ are the spatial coordinates in the $i$ th and $j$ th directions $(\mathrm{m}) . i, j$ denote the three directions in the global Cartesian coordinate system. $F$ is the external forces acting on the fluid except for gravitation. The Lorentz force resulting from EMS is added through this item $F$.

Equation of continuity is:

$$
\frac{\partial u_{j}}{\partial x_{j}}=0
$$

The realizable $k-\varepsilon$ turbulence model is used to treat the turbulence characteristics of the fluid flow, which is shown below:

$$
\begin{aligned}
\rho \frac{\partial k}{\partial t}+ & \rho \frac{\partial\left(k u_{j}\right)}{\partial x_{j}}=\frac{\partial}{\partial x_{j}}\left[\left(\mu_{0}+\frac{\mu_{t}}{\sigma_{k}}\right) \frac{\partial k}{\partial x_{j}}\right]+G_{k}+G_{b}-\rho \varepsilon+S_{k} \\
& \rho \frac{\partial \varepsilon}{\partial t}+\rho \frac{\partial\left(\varepsilon u_{j}\right)}{\partial x_{j}}=\frac{\partial}{\partial x_{j}}\left[\left(\mu+\frac{\mu_{t}}{\sigma_{\varepsilon}}\right) \frac{\partial \varepsilon}{\partial x_{j}}\right] \\
& +\rho C_{1} S \varepsilon-\rho C_{2} \frac{\varepsilon^{2}}{k+\sqrt{v \varepsilon}}+C_{1 \varepsilon} \frac{\varepsilon}{k} C_{3 \varepsilon} G_{b}+S_{\varepsilon}
\end{aligned}
$$


where $C_{1}=\max \left[0.43, \frac{\eta}{\eta+5}\right], \eta=S \frac{k}{\varepsilon}, S=\sqrt{2 S_{i j} S_{i j}}$.

In these equations, $G_{k}$ represents the generation of turbulence kinetic energy due to the mean velocity gradients. $G_{b}$ is the generation of turbulence kinetic energy due to buoyancy. $C_{2}$ and $C_{1 \varepsilon}$ are constants. $\sigma_{k}$ and $\sigma_{\varepsilon}$ are the turbulent Prandtl numbers for the turbulent kinetic energy $k$ and the dissipation rate of turbulent kinetic energy $\varepsilon$, respectively. $S_{k}$ and $S_{\varepsilon}$ are user-defined source terms.

This turbulence model differs from the standard $k-\varepsilon$ model in the following two aspects: 1) the realizable $k-\varepsilon$ model contains a new formulation for the turbulent viscosity; 2) a new transport equation for the dissipation rate has been derived from an exact equation for the transport of the mean-square vorticity fluctuation. The term "realizable" means that the model satisfies certain mathematical constraints on the Reynolds stresses, consistent with the physics of turbulent flows. The benefit of the realizable $k-\varepsilon$ model is to predict accurately the spreading rate of both planar and round jets. It provides superior performance for flows involving rotation, boundary layers under strong adverse pressure gradients, separation and recirculation. This model has been extensively validated for a wide range of flows, ${ }^{17)}$ including rotating homogeneous shear flows, free flows including jets and mixing layers, channel and boundary layer flows, and separated flows. For all these cases, the performance of the model has been found to be substantially better than that of the standard $k-\varepsilon$ model. Especially, the realizable $k-\varepsilon$ model resolves the round-jet anomaly, i.e., it predicts the spreading rate for axis-symmetric jets as well as that for planar jets. More mathematical details of this model can be referenced in the ANSYS-FLUENT manuals. Thereby, the realizable $k-\varepsilon$ model is considered to be suitable for the prediction of rotational flow driven by electromagnetic field. Still, the standard wall function model is used to treat the turbulent boundary layer. The convergence conditions for the flow variables are set to $10^{-4}-10^{-6}$.

\subsection{Lorentz Force Model}

The complexity in this simulation is the EMS driven flow phenomenon. As is known, the rotational electromagnetic field generated by the EMS coils induces an electric current in the conductive steel melt. The interaction of the electric current with the magnetic field produces a Lorentz force acting on the fluid which carries the current. The Lorentz force strives to brake the relative movement between the fluid and the magnetic field. For the rotational EMS field, the Lorentz force drives the fluid to flow following the rotational direction of the electromagnetic field. The Lorentz force can be calculated by first solving Maxwell's equations and then applying Ohm's law as is done by the previous works. ${ }^{18-22)}$ In this paper, instead of solving the complicated Maxwell's equations, a semi-empirical equation is proposed to calculate the Lorentz force in time-averaged scheme:

$$
\begin{gathered}
F_{x}=-\frac{1}{2} c_{1} \sigma\left(f_{m} \alpha B_{0}\right)^{2}\left(U_{x}-2 \pi f y\right) \\
F_{y}=-\frac{1}{2} c_{1} \sigma\left(f_{m} \alpha B_{0}\right)^{2}\left(U_{y}+2 \pi f x\right) \\
F_{z}=-\frac{1}{2} \sigma\left(f_{m} \alpha B_{0}\right)^{2} U_{z} \\
\alpha=1+\frac{\pi}{2} c_{0} \mu_{0} \sigma f R^{2}\left(1+\frac{U_{t}}{2 \pi f r}\right) \ldots
\end{gathered}
$$

where $U_{t}=U_{y} \frac{x}{r}-U_{x} \frac{y}{r}, r=\sqrt{x^{2}+y^{2}}, R=2 \sqrt{\frac{a b}{\pi}}$. In these equations, $F_{x}, F_{y}, F_{z}$ are the Lorentz force in the three directions, $\mathrm{Nm}^{-3} ; U_{x}, U_{y}, U_{z}$ are fluid flow velocity, $\mathrm{ms}^{-1} ; \sigma$ is electrical conductivity, $714000 \mathrm{~S} / \mathrm{m}$. $f_{m}$ is a distribution function of the measured magnetic field along the mould center; $B_{0}$ is the flux density of the magnetic field at the stirring center based on in-house measurement as shown in Figs. 1 and 9, T; $f$ is the stirring frequency, $\mathrm{Hz} ; \mu_{0}$ is magnetic permeability, $1.256637614 \times 10^{-6} \mathrm{Hm}^{-1} ; U_{t}$ is tangential velocity, $\mathrm{ms}^{-1} ; \mathrm{x}, \mathrm{y}, \mathrm{z}$ are coordinate points in the calculation domain and $(0,0, \mathrm{z})$ is the central axis of the rotational electromagnetic field, m. a and b are the half sizes of the bloom/ billet, $\mathrm{m} ; c_{0}$ and $c_{1}$ are coefficients, $c_{0}, c_{1}<1$.

In addition, Joule heating $Q\left(\mathrm{~W} / \mathrm{m}^{3}\right)$ is also included with the following proposed model:

$$
Q=\frac{1}{2} c_{2} \sigma\left(f_{m} \alpha B_{0}\right)^{2}\left[\left(U_{x}-2 \pi f y\right)^{2}+\left(U_{y}+2 \pi f x\right)^{2}+U_{z}^{2}\right] \ldots
$$

where $c_{2}$ is coefficient. This method can save large amount of computation time and effort being used on the grid operation between electromagnetic field and fluid flow field. Thus, the computation efficiency can be much improved for the complicated magneto-hydrodynamic phenomenon in the casting process, meanwhile representing major rotational flow features under the action of EMS.

Since the stirred flow usually results in severe surface deformation due to the upward stream along the mould corners, the free surface phenomenon at the steel melt/slag layer is also simulated by the volume of fluid (VOF) method. The predicted surface wave can be compared with the oscillation marks left on the bloom/billet surface to validate the models and methods being used in the simulation.

\subsection{Inclusions Tracking}

After the flow field is computed, particle trajectories are simulated to capture the movement of inclusions inside the steel melt due to the difference of densities from the steel melt and the difference of inclusion sizes. The inclusions in the melt can float upwards being trapped by the liquid slag, or flow downwards with the fluid into the deeper strand pool being merged in the solidified structure. To simplify the computation, the reflection boundary condition is used on the strand shell. This is to say that when an inclusion touches the solidified strand shell, it is bounced back into the melt. Only two destinations are considered in the calculation, being removed by the slag layer or flowing out to the deeper pool and included into the solidified shell. In order to calculate the transient movement of inclusions in the steel flow, the Euler-Lagrange approach was used and the equation is as below:

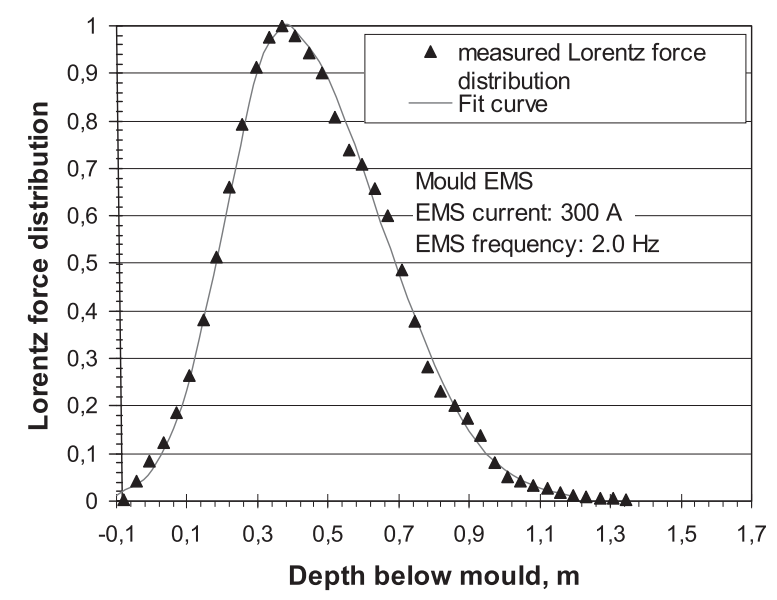

Fig. 9. Measured Lorentz force distribution in the bloom mould (Ovako Imatra). 


$$
\begin{aligned}
& \frac{d U_{\mathrm{p}}}{d t}=F_{\mathrm{D}}\left(U-U_{\mathrm{p}}\right)+\frac{\rho_{\mathrm{p}}-\rho}{\rho_{\mathrm{p}}} g \\
& +\frac{\rho}{2 \rho_{\mathrm{p}}} \frac{d}{d t}\left(U-U_{\mathrm{p}}\right)+\frac{\rho}{\rho_{\mathrm{p}}} U_{\mathrm{p} i} \frac{\partial U}{\partial x_{i}}
\end{aligned}
$$

Here, $U_{\mathrm{p}}$ is the moving velocity of a particle in the melt $\left(\mathrm{ms}^{-1}\right) ; \rho_{\mathrm{p}}$ is density of the particle $\left(\mathrm{kgm}^{-3}\right)$. The left hand of the equation is the acceleration term of the particle to be calculated. The first term on the right hand is the drag force. The second term is the buoyancy term. The third term is the virtual mass force, which represents an additional resistance due to the attached, "virtual" fluid mass around the particle in the process of acceleration movement. The fourth term $\frac{\rho}{\rho_{\mathrm{p}}} U_{\mathrm{p} i} \frac{\partial U}{\partial x_{i}}$ is the action force of a shear flow field on the particle, which is resulted from a gradient of the fluid velocities around the particle and the direction of which is opposite to that of the velocity gradient. In a rotational flow field, this force supplies a pushing force pointing to the rotation center to drive the particle to move with the fluid rotationally. In this context, a centripetal force is not needed to impose on the particle, even though it rotates in the fluid, since the fourth term has already played this role. Turbulence treatment on the interaction of the particle with turbulent eddies in the fluid can be referenced in Trindade et al. ${ }^{23)}$

Due to the random nature in the trajectory calculations resulting from the turbulence treatment, one trajectory cannot be reproduced by another even with all the same computation parameters. However, by injecting a large number of particles through the SEN into the mould, statistic results can give a clear trend to show the percentage of the inclusions which are trapped by the slag layer. The particle diameter ranges from 1 to 200 microns so that the effect of flow field on almost all sizes of inclusions can be demonstrated. Thereafter, the influence of the different SEN designs on inclusions can be evaluated.

\section{Modeling Results}

\subsection{Flow Field and Validation with a Billet Casting}

The rotational flow field is simulated for the billet casting with three EMS settings of $0 \mathrm{~A}$ (no stirring), $150 \mathrm{~A}$, and 310 A electric current, as shown in Fig. 10. When stirring is not applied (Fig. 10(a)), the liquid stream straightly penetrates deep into the strand pool. At 150 A stirring intensity (Fig. 10(b)), the rotation flow of the steel melt is observed, the maximum velocity of which is around $0.35 \mathrm{~m} / \mathrm{s}$ in the near wall region of the stirring center. Using again Takahashi et al. expression (Eq. (1)), the corresponding dendrite deflecting for this calculated liquid flow would be $25^{\circ}$, which is little higher than the measured values. The reason might be that the maximum tangential velocity exists only for a short while at the solidification front with the continuous downward movement of the strand shell.

An interesting phenomenon in Fig. 10(b) is that the incoming stream is declined towards the back wall of the strand shell under the interaction of the horizontal shear flow field in the rotational flow with the vertical shear flow field of the injecting stream. Simultaneously, penetration depth is decreased due to the dissipating effect of the rotational flows on the incoming stream. The declined injecting stream may result in non-uniform thermal distribution on the strand shell facing the stream, and if more seriously, may lead to vertical central cracks and even break-out.

When the stirring intensity is increased to $310 \mathrm{~A}$, the incoming stream is straightened and shortened under the action of the strengthened shear flow field of the horizontal

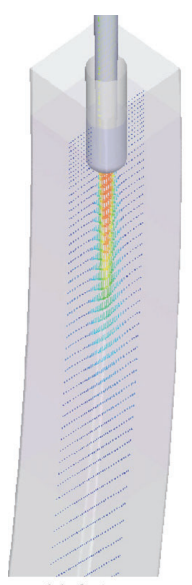

(a) $0 \mathrm{~A}$

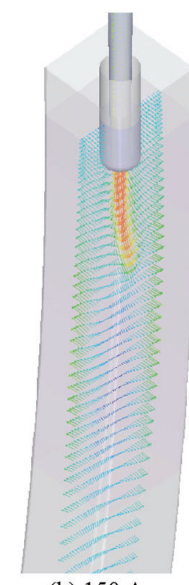

(b) $150 \mathrm{~A}$

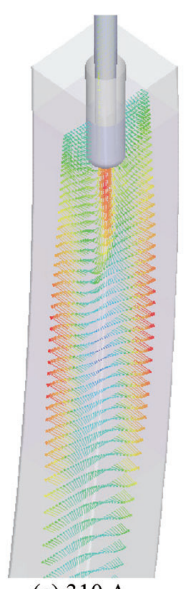

(c) $310 \mathrm{~A}$

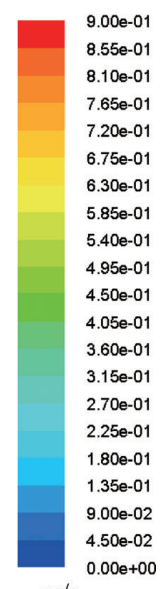

$\mathrm{m} / \mathrm{s}$
Fig. 10. Simulated flow field in the billet mould with different EMS intensities (0, 150, $310 \mathrm{~A})$.

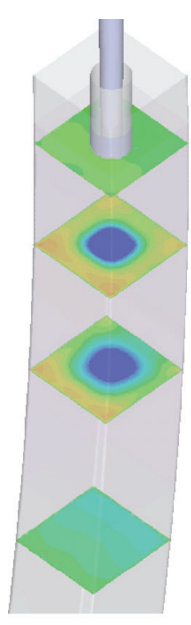

(a) unstirred

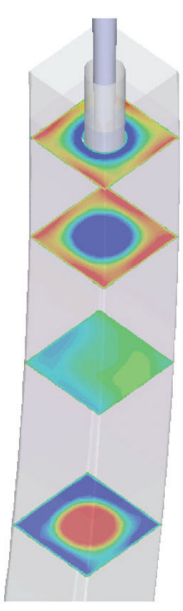

(b) stirred

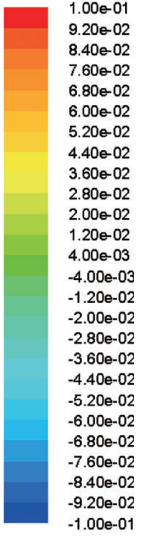

$\mathrm{m} / \mathrm{s}$
Fig. 11. Vertical component of the fluid flow velocities on horizontal sections of 40, 300, 584 (stirring center), and 1000 $\mathrm{mm}$ below meniscus. Red represents upward flow; blue represents downward flow. (a) Unstirred; (b) Stirred with 310 A.

rotation, see Fig. 10(c). The maximum tangential velocity is around $0.8 \mathrm{~m} / \mathrm{s}$ in the stirring central zone. It can be seen that correct matching of the horizontal rotation flow with the vertical injection flow in the mould is significant for casting qualities, which is related to casting speed, geometrical design and stirring parameters.

To validate the flow field especially the upward corner flows, vertical components of the flow field are presented as in Fig. 11 for unstirred (a) and stirred $(310$ A) (b) cases. Upward corner flows are observed for stirred case with the magnitude of $0.10-0.22 \mathrm{~m} / \mathrm{s}$ along the corners above the stirring center, and in most of the corner length the upward flow is between $0.15-0.20 \mathrm{~m} / \mathrm{s}$, which is over predicted compared with the casting experiments evaluated by dendrite deflections in the corner region where $0.14 \mathrm{~m} / \mathrm{s}$ was measured (see Section 2.4). A horizontal distribution of the maximum vertical velocity above the stirring center was shown in Fig. 7(b) for comparison with the measured results. Naturally, the modeling results above the stirring center are higher than the measured results on the average meaning. Generally, the modeling results agree with the experiments in the flow pattern and trend. It is noteworthy that below the stirring center, corner flows are converted to 
downward directions and the central flow converted to upward, just opposite to the volumetric flow pattern above the stirring center. Besides, on the stirring central plane, the vertical velocity component is relatively uniform (Fig. 11(b)), demonstrating a plug flow feature to the downward direction. However, considering the tangential components, as can be seen in Fig. 10(c), majored by a horizontal rotation flow, the plug flow on the stirring central plane is actually a rotational plug flow toward the downward direction.

Resulting from the upward flows at the mould corners, the raised free surface waves were also predicted in the simulation as can be seen in Fig. 12. The wave height was $10 \mathrm{~mm}$ at the corners above the average meniscus level. This is in agreement with the measured oscillation marks as shown in Fig. 5(a). Because of the simplification on the model assumptions and limitation of VOF method, the discontinuity phenomenon of the oscillation marks at the corner cannot be exactly represented by the present modeling. However, the asymmetry of the surface wave at the corners can be clearly predicted in the contour map as shown in Fig. 12. Considering initial solidification, this discontinuity can be explained that the impinging momentum pushed the thin shell wafer to a closer contact with the counterpart corner wall and enhances the initial solidification on this side of the corner, so as to form a discontinued, or say, uneven wafer shell on the connected two sides of the corners.

Another interesting phenomenon from the modeling results (Fig. 12) is that four vortexes appeared on the free surface around the SEN tube, geometrically corresponding to the four corners or four side walls. The reason might be owing to the negative pressure caused by the non-uniform tangential circulation flows around the shroud wall affected by the square or rectangular geometric feature of the mould. Another reason, which also contributes to deepen these vortexes, is that the "pulling" effect of the injection stream causes pressure drop to the free surface above. These vortexes may re-entrain the slag droplets or trapped inclusions in the slag layer back into the steel melt and deteriorate cleanliness. By reducing stirring intensity and deepening immersion depth, these vortexes can be relieved.

\subsection{Effect of Different SEN Designs on the Free Menis- cus for Bloom Casting}

Figure 13 demonstrates the free meniscus influenced by the different SEN designs in the bloom casting process. The red color at the mould corners represents the raised wave above the average meniscus level, and the blue color around
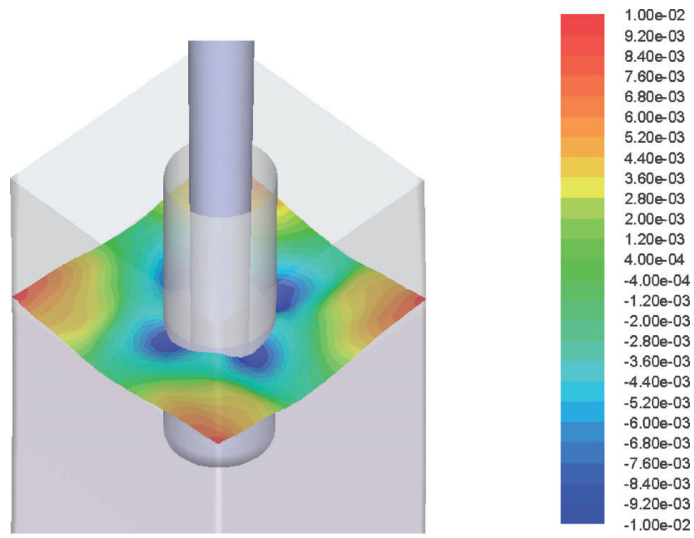

Fig. 12. Free surface wave in the billet casting with 310 A EMS. The surface is raised up by $10 \mathrm{~mm}$ at the corners which is in good agreement with the measured oscillation marks at the corners. the tube represents the vortexes below the average meniscus level. The meniscus wave at the corners is mainly driven by upward corner flows caused by the rotational flow field as illustrated in the billet simulation (Fig. 11(b)). From Fig. 13, it is seen that the influence of different SENs on the free surface wave is little on the general free meniscus configuration.

\subsection{Effect of Different SEN Designs on Inclusion Removal}

Inclusion tracking is performed for the bloom casting with various SEN designs. By tracking the trajectories of a large number of particles, herein 10000 in the calculation, statistic results of inclusion removal rate are obtained, as shown in Fig. 14. The curves in Fig. 14 indicate that ported SENs give better removal rate than the straight bore metering SEN. Especially, the 2-port SEN with guiding angle of upward $15^{\circ}$ shows the best result. The 4-port SEN does not give the best result as expected. Generally speaking, it is clear from the simulation results that ported SENs show obvious advantage in cleanliness over the metering SEN and the 4-ported SEN does not show superior to the 2-port SENs.

\subsection{Effect of Casting Speed on Inclusion Removal}

Casting speed is one of the main factors influencing the removal rate of inclusions, since it directly determines the flow field magnitude in the mould, and also the residence time of steel melt through the mould. Figure 15 illustrates the influence of casting speed on the inclusion removal, by using (a) the straight bore SEN and (b) the 2-port SEN with

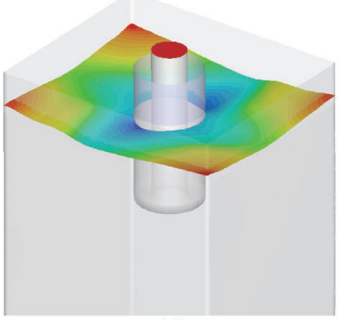

(a)

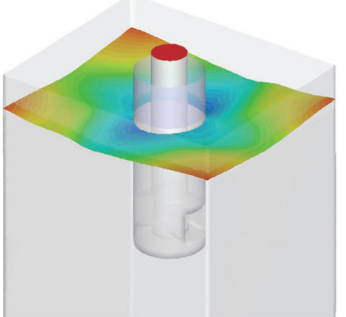

(c)

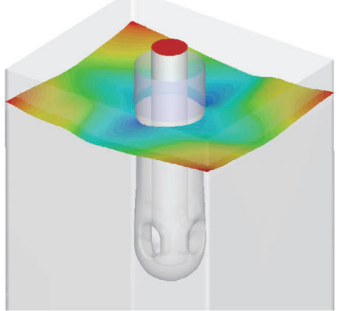

(e)

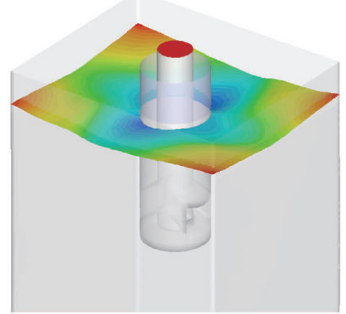

(b)

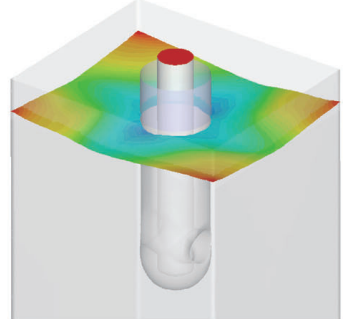

(d)

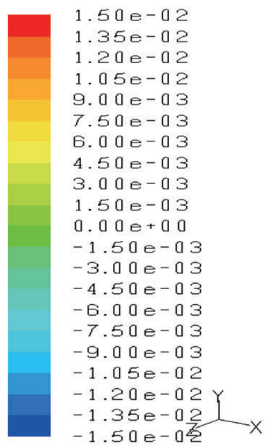

$\mathrm{m}$

Fig. 13. The free meniscus waves with various SEN designs. 


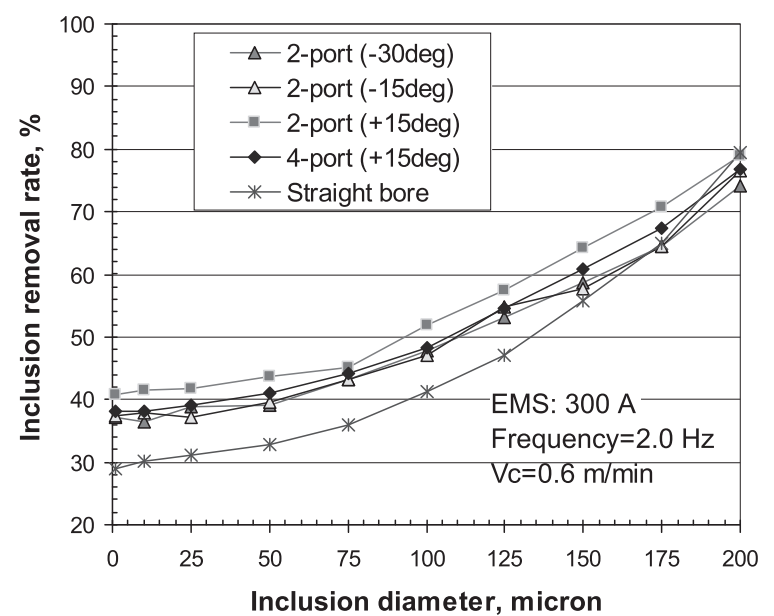

Fig. 14. Inclusion removal rate with various SEN designs.

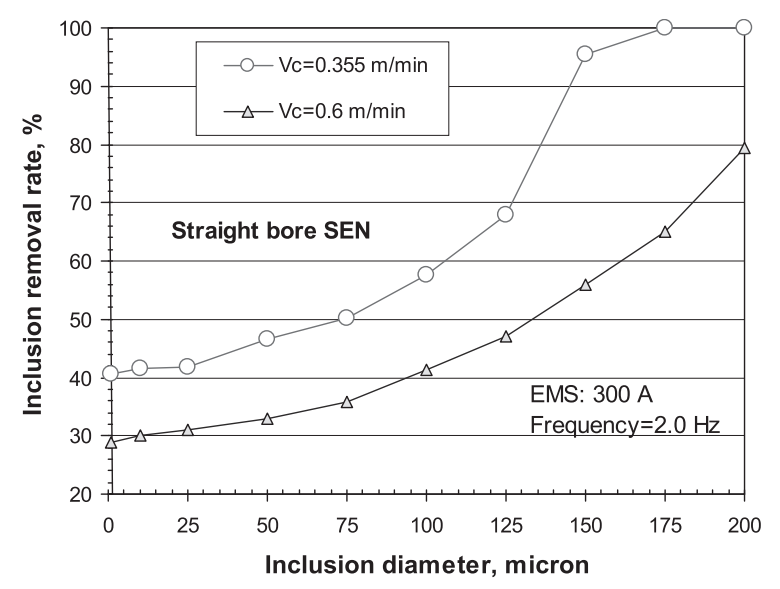

(a) straight bore SEN

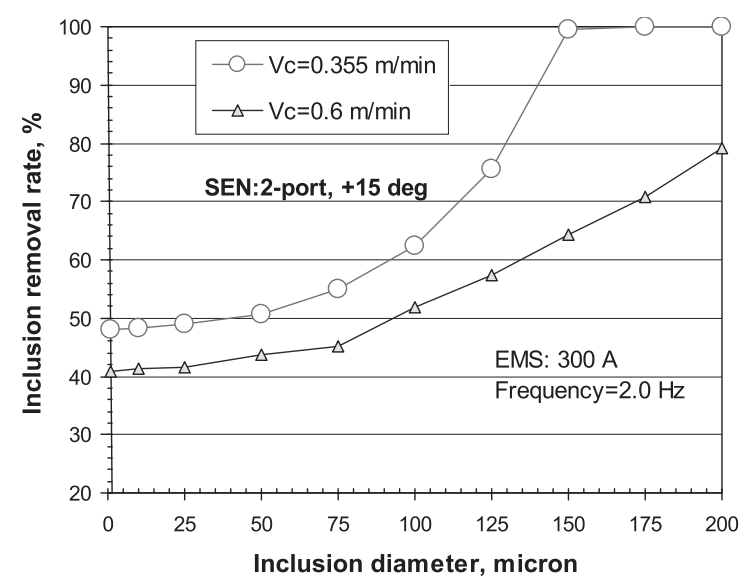

(b) 2-port SEN

Fig. 15. Casting speed substantially influences the inclusion removal rate.

the guiding angle of upward $15^{\circ}$. When the casting speed was reduced from $0.6 \mathrm{~m} / \mathrm{min}$ to $0.355 \mathrm{~m} / \mathrm{min}$, the removal rate was increased substantially. This is also to say that reducing casting speed is an efficient way to increasing cleanliness if productivity allows.

From another side, this example also indicated that increasing casting speed brings about very stringent technical demands for cleanliness control owing to the reduced residence time of the steel melt through the mould. With this understanding, it can be deduced that larger product section

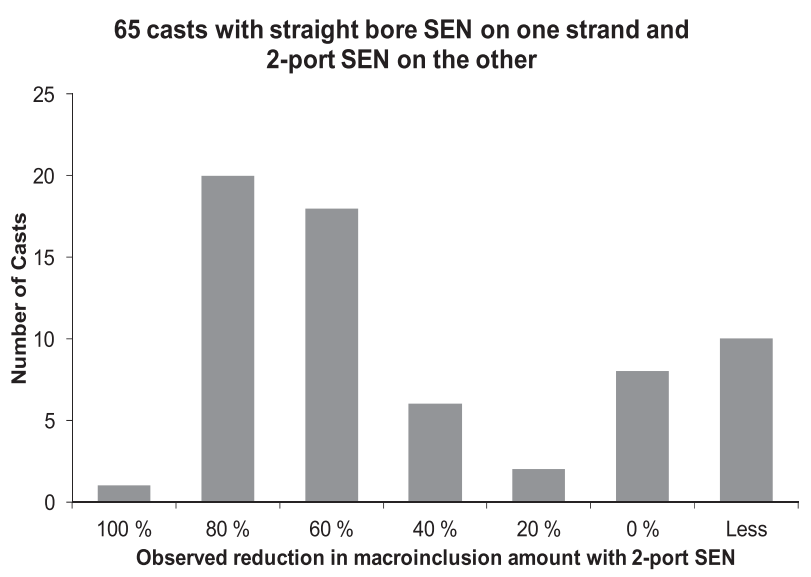

Fig. 16. Casting trials show the improvement of inclusion removal with 2-port SEN compared with the straight bore SEN (Ovako).

with lower casting speed is favorable for cleanliness, supposing the productivity is maintained on the same level. This is conflicting to the concept and practice of near net shape casting developed and applied in the recent three decades. ${ }^{24)}$ Another option is the multi-strand caster arrangement with an appropriate casting speed to compromise cleanliness, sizes and productivity. Anyhow, metallurgical engineering marches ahead this way, depending on practical needs, technological capabilities and economical motivations.

\section{Casting Trials with Two-port SEN and Metering SEN on a Bloom Caster}

Based on the modeling results obtained for the bloom casting, plant trials were carried out on a two-strand bloom caster with $75 \mathrm{t}$ heat size, $15 \mathrm{~m}$ radius, stopper rod control and M-EMS. The CFD modeling showed the best cleanliness results for the 2-port SEN design with guiding angle upward $15^{\circ}$. However, due to practical reasons, the 2-port SEN design with the guiding angle downward $15^{\circ}$ was chosen for the plant trials.

In the trial campaign a straight bore SEN was used on one strand, and the 2-port SEN with downward $15^{\circ}$ guiding angle on the second strand. Altogether 65 heats were cast in the trials with varying steel grades: quenching and tempering steels, boron steels, engineering steels, case hardening steels, micro-alloyed steels and spring steels.

Sampling of the heats was carried out at the rolling mill from the hot rolled bars. Samples were obtained from both strands at the beginning and in the middle of the heat. Sample length varied between 210 to $500 \mathrm{~mm}$ depending on the bar diameter. Non-metallic inclusion content was evaluated with immersion ultrasound method. In the method the steel samples were machined to give them a $90 \times 90 \mathrm{~mm}$ or less cross-section, again depending on the sample diameter. A spherical ultrasonic transducer with a $20 \mathrm{~mm}$ diameter and a $10 \mathrm{MHz}$ frequency was used for scanning the sample perpendicular to the casting direction.

For each heat, the cleanliness results were compared between the 2-port SEN and the straight bore SEN. The comparison yielded the result in Fig. 16. Of the 65 heats, 10 heats showed that 2-port SEN influenced cleanliness negatively while 8 heats showed no influence and 47 heats showed improvement in cleanliness. Particularly, of the 47 heats with positive results, 39 casts indicated over $60 \%$ reduction in the non-metallic inclusion amount. This is a significant improvement in the cleanliness of the product. 


\section{Summary Remarks}

Comprehensive billet casting trials were carried out to study the effect of magnetohydrodynamic phenomena induced by EMS on the flow field and the solidification microstructures. It was found that: 1) application of EMS did not cause sensible disturbance to the meniscus stability; 2) EMS induced rotational flow resulted in a rise of oscillation marks at the mould corners due to the upward flow streams along the mould corners; 3 ) the sustained upward flow streams along the mould corners led to downward deflected dendrite morphology at the billet corners; 4) there appeared to be a discontinuity of the oscillation marks at the intersecting place of the connected walls, which was formed by the impinging effect on one side of the corner by the rotational flow; 5) EMS clearly increased central equiaxed zone from $10-15 \%$ in the non-stirring case to over $45 \%$ with the 310 A stirring intensity; 6) rhomboidity was observed to relate to the unsteady flow in the mould during changes of casting speed and EMS intensity.

Using CFD method, EMS induced rotational flow phenomenon and near meniscus phenomenon in a billet casting were simulated and validated with casting trial experiments. Simulated corner flow agreed well with the measured result evaluated from dendrite deflections, and the free surface wave coincided with the measured oscillation marks. From the modeling results, a declining phenomenon of the injected stream from the SEN was observed towards the back wall under certain stirring intensities. This would influence thermal distribution of the strand shell and casting qualities, thus requiring correct matching of the vertical shear flow field with the horizontal rotation flow in order to obtain favorable flow field. In addition, vortexes were found on the free surface around the SEN tube, which were influential to the cleanliness of steel melt.

Through modeling the free meniscus and inclusion trajectories for a bloom casting with various SEN designs, their influence on the cleanliness was evaluated. It was found that ported SENs demonstrated obvious advantage on inclusion removal over the conventional straight bore SEN. The 4port SEN did not give better results than the 2-port SENs. Casting trials with 65 bloom casting heats verified the modeling results and showed remarkable improvement in cleanliness by using a 2-port SEN. Reducing casting speed is also an effective way to improve cleanliness because of the increased residence time of the steel melt through the casting mould.

In general, application of magnetohydrodynamic techniques greatly changes the volumetric flow pattern in the mould and deeper strand pool, and remarkably impacts the microstructural morphology, segregation, soundness, cleanliness and even properties of the cast products. Geometric constraint directly controls the fluid boundary and injecting guidance in the upper mould and sensibly influences surface quality, even subsurface quality, and cleanliness. Computational modeling has become an essential and indispensable assisting technique in fluid control engineering and designs. It supplies quantitative solutions, macroscopic/microscopic descriptions, process representations and optimizations for a complicated fluid flow system. In addition, it can also reveal inaccessible and even unrevealed phenomena with proper models as shown above. These experimental and modeling results stress again the importance of mould flow patterns and comprehensive fluid control techniques in the pursuit of ever cleaner steels and better cast qualities.

\section{Acknowledgment}

The authors appreciate EU MAGNETO-HYDRO project (RFCS-CT-2007-00012) for the financial support. The authors would like to thank Dr. Shahid Riaz, Tata Steel (UK), for valuable discussions and supplying numerous casting cases during the project. The authors would also thank Gerdau R\&D and Ovako Imatra Oy Ab for supplying the casting trial data and permission for publishing the results.

\section{REFERENCES}

1) A. D. Wilson: Proc. of Robert I. Jaffee Memorial Symp. on Clean Materials Technology, ed. by R. Visvanathan, ASM/TMS, Warrendale, PA, (1992), 135

2) K. H. Mayer and C. Berger: Proc. of Clean Steel: Super Clean Steel, The Institute of Materials, London, (1995), 195.

3) L. E. K. Holappa: Proc. of 6th Int. Iron and Steel Congr., Vol. 3, ISIJ, Tokyo, (1990), 494.

4) P. Jönsson, M. Andersson, L. Jonsson, F. Reinholdsson and G. Runnsjö: Proc. of Non-metallic Inclusions Control and Continuous Improvement of Processes Based on Objective Measurement, Jernkontorets Forskning D801, Stockholm, (2004), 1.

5) P. Ölund: Control of Non-metallic Inclusion Composition and Production of Clean Steels, ed. by K. Nakajima, P. Jönsson and A. Karasev, Vol. 1, Royal Institute of Technology, Stockholm, (2011), 9.

6) E. Torres-Alonso, R. D. Morales, S. Garcia-Hernandez, A. NajeraBastida and A. Sandoval-Ramos: Metall. Mater. Trans. B, 39B (2008), 840.

7) A. Sormann, E. Hanse and A. Vaterlaus: Research Report of The Institute of Materials, Steel Division, The Royal Society, London, (1998), 1.

8) H. Nakata, J. Tsubokura and H. Takahashi: Proc. of 6th Int. Iron and Steel Congr., Vol. 3, ISIJ, Tokyo, (1990), 470.

9) S. Yokoya, S. Takagi, M. Kaneko, M. Iguchi, K. Marukawa and S. Hara: ISIJ Int., 41 (2001), No. 10, 1215.

10) S. Kholmatov, S. Takagi, L. Jonsson, P. Jönsson and S. Yokoya: Steel Res. Int., 79 (2008), No. 1, 31.

11) S. Kholmatov, S. Takagi, L. Jonnsson, P. Jönsson and S. Yokoya: ISIJ Int., 47 (2007), No. 1, 80.

12) S. Wang, S. Louhenkilpi and P. Väyrynen: Proc. of 3rd Steelsim Conf., ASMET, Leoben, (2009), 180.

13) J. Pindor and K. Michalek: Proc. of 7th Int. Conf. on Clean Steel, Hungarian Mining and Metallurgical Society, Balatonfüred, (2007), 246.

14) T. Takahashi, K. Ichikawa, M. Kudou and K. Shimamura: Tetsu-toHagané, 61 (1975), 2198.

15) J. Madias: Proc. of 5th Int. Congr. on the Science and Technology of Steelmaking, Institute of Iron and Steel Technology, Dresden, (2012), 1.

16) P. E. Ramirez-Lopez, K. C. Mills, P. D. Lee and B. Santillana: Proc. of 7th ECCC, METEC, Düsseldorf, (2011), Session 8, 1.

17) T.-H. Shih, W. W. Liou, A. Shabbir, Z. Yang and J. Zhu: Comput. Fluid., 24 (1995), No. 3, 227.

18) K.-H. Spitzer, M. Dubke and K. Schwerdtfeger: Metall. Trans. B, 17B (1986), 119

19) J. L. Meyer, N. EI-Kaddah, J. Szekely, C. Vivés and R. Ricou: Metall. Trans. B, 18B (1987), 529.

20) H. Yamamura, T. Toh, H. Harada, E. Takeuchi and T. Ishii: ISIJ Int., 41 (2001), No. 10, 1229.

21) K. Cukierski and B. G. Thomas: Metall. Mater. Trans. B, 39B (2008), 94.

22) M. J. Cho, E. B. Park and S. W. Kim: ISIJ Int., 50 (2010), No. 8, 1180 .

23) L. B. Trindade, J. E. A. Nadalon, A. C. F. Vilela, M. T. Vilhena and R. B. Soares: Steel Res. Int., 78 (2007), No. 9, 708.

24) L. E. K. Holappa and S. Wang: Proc. of Sohn Int. Symp. on Advanced Processing of Metals and Materials, Vol. 2, TMS, Warrendale, PA, (2006), 455. 